\title{
THE RIEMANN ZETA AND ALLIED FUNCTIONS
}

\section{SARVADAMAN CHOWLA}

1. On $\zeta(s)$. Let $s$ be a complex variable, $s=\sigma+i t$, where $\sigma$ and $t$ are real. We define

$$
\zeta(s)=\sum_{1}^{\infty} n^{-s} \quad(\sigma>1) .
$$

For $\sigma>1$ it is easily deduced from (1) that

$$
\pi^{-s / 2} \Gamma\left(\frac{s}{2}\right) \zeta(s)-\frac{1}{s(s-1)}=\int_{1}^{\infty}\left(x^{8 / 2-1}+x^{(1-s) / 2-1}\right) \Theta(x) d x
$$

where

$$
\Theta(x)=\sum_{n=1}^{\infty} e^{-n \pi x}
$$

To prove (2) we use Jacobi's relation

$$
1+2 \Theta(x)=\frac{1}{x^{1 / 2}}\left\{1+2 \Theta\left(\frac{1}{x}\right)\right\} .
$$

Since the right-hand side of (2) is an analytic function of $s$ for all complex values of $s$, we use (2) to define $\zeta(s)$ over the whole $s$-plane.

It easily follows from (2) that

$$
\begin{aligned}
& \zeta(s)-\frac{1}{s-1} \text { is an integral function of } s \\
& \quad \pi^{-s / 2} \Gamma\left(\frac{s}{2}\right) \zeta(s)=\pi^{-(1-s) / 2} \Gamma\left(\frac{1-s}{2}\right) \zeta(1-s)
\end{aligned}
$$

We have

$$
\zeta(s)=\prod_{p}\left(1-p^{-s}\right)^{-1} \quad[\sigma>1],
$$

where $p$ runs through all primes. Equation (5) follows immediately from the so-called unique factorization theorem of arithmetic, which states that a positive integer can be decomposed into a product of primes in one and only one way.

An address delivered before the Annual Meeting of the Society at New York on December 28, 1949 by invitation of the Committee to Select Hour Speakers for Annual and Summer Meetings; received by the editors December 23, 1950 and, in revised form, December 5, 1951. 
Equation (4) is the "functional equation" of the zeta function due to Riemann. From (3), (4), and (5) it easily follows that except for the "trivial" zeros of $\zeta(s)$ at $s=-2,-4,-6, \cdots$, all the remaining zeros of $\zeta(s)$ lie in the critical strip $0<\sigma<1$. In fact it also follows from the Hadamard theory of integral functions that there is an infinity of complex zeros in this strip.

The "Riemann hypothesis," one of the most famous and difficult of unsolved problems today, states that all the nonreal zeros $s$ of $\zeta(s)$ satisfy $\sigma=1 / 2$. It can also be stated as follows:

If $1^{-s}-2^{-s}+3^{-s}-4^{-s}+\cdots \cdots=0(0<\sigma<1)$, then $\sigma=1 / 2$. This follows from the fact that

$$
\left(1-2^{1-s}\right) \zeta(s)=1^{-s}-2^{-s}+3^{-s}-4^{-s}+-\cdots
$$

is clearly true for $\sigma>1$, and hence for $\sigma>0$.

Riemann also found that the zero $s$ of $\zeta(s)$ with the smallest ordinate $t=\alpha_{1}$ satisfies

$$
s=1 / 2+14.1386 \cdots i=1 / 2+\alpha_{1} i .
$$

A later calculation by Gram showed that $\alpha_{1}=14.1347 \cdots$. Let $\alpha_{n}$ run through all the solutions $\alpha$ of $\zeta(1 / 2+i \alpha)=0$; then (assuming the Riemann hypothesis) we have

$$
\begin{aligned}
\sum_{n=1}^{\infty}\left(\alpha_{n}^{2}+\frac{1}{4}\right)^{-1} & =1+\frac{1}{2} C-\frac{1}{2} \log \pi-\log 2 \\
& =.02309570896612103381
\end{aligned}
$$

according to a calculation of Riemann. Further zeros of $\zeta(s)$ have been calculated by Backlund, Hutchinson, and Titchmarsh. In particular Titchmarsh calculated all complex zeros $s$ with $|t| \leqq 1468$. They all had $\sigma=1 / 2$, in confirmation of the Riemann hypothesis. Rademacher and Lehmer hope to substantially extend the range of these calculations. Should these or any authors find a zero off the line $\sigma=1 / 2$, such a discovery would certainly be sensational. It would, however, be a sad thing for mathematics, as it would destroy in one cruel blow the beautiful and harmonious structure which has been built on the assumption of the Riemann hypothesis.

2. On $\zeta(s)$, continued. We owe to A. Selberg the sharpest known estimate on the number of zeros $s$ of $\zeta(s)$ with $\sigma=1 / 2,0 \leqq t \leqq T$. Call the number of such zeros $N_{0}(T)$. Hardy was the first to prove in 1915 that

$$
N_{0}(T) \rightarrow \infty \quad[T \rightarrow \infty] .
$$


Later Hardy and Littlewood sharpened this estimate to

$$
N_{0}(T)>c T \text {, }
$$

where $c$ is a positive constant independent of $T$. Selberg proved that

$$
N_{0}(T)>c T \log T \text {. }
$$

Denote by $N(T)$ the number of zeros $s$ of $\zeta(s)$ with $0<\sigma<1,0 \leqq t \leqq T$. $H$. von Mangoldt proved Riemann's conjecture that

$$
N(T) \sim(1 / 2 \pi) T \log T .
$$

From the last two relations

$$
\frac{N_{0}(T)}{N(T)}>c
$$

In his 1943 paper A. Selberg proved more; namely, he proved the following:

Let $U \geqq T^{a}$, where $a>1 / 2$; then there is an $A=A(a)>0$ and a $T_{0}=T_{0}(a)$ such that

$$
N_{0}(T+U)-N_{0}(T)>A U \log T
$$

(p. 46 of Selberg).

Let $p_{n}$ denote the $n$th prime. Erdös has conjectured that

$$
\sum_{p_{n} \leqq x}\left(p_{n}-p_{n-1}\right)^{2}=O(x \log x) .
$$

This would imply

$$
\sum_{p_{n} \leqq x} \frac{\left(p_{n}-p_{n-1}\right)^{2}}{p_{n}}=O\left(\log ^{2} x\right) .
$$

On the Riemann hypothesis A. Selberg proved that

$$
\sum_{p_{n} \leqq x} \frac{\left(p_{n}-p_{n-1}\right)^{2}}{p_{n}}=O\left(\log ^{3} x\right) .
$$

Denote by $N(T)$ the number of zeros $\beta+i \gamma$ of $\zeta(s)$ for which $0<\gamma<T$, if $T$ is not equal to any $\gamma$; otherwise we put

$$
N(T)=\lim _{\epsilon \rightarrow 0} \frac{1}{2}\{N(T+\epsilon)+N(T-\epsilon)\} .
$$

It is well known that

$$
N(T)=\frac{T}{2 \pi} \log \frac{T}{2 \pi}-\frac{T}{2 \pi}+\frac{7}{8}+S(T)+O\left(T^{-1}\right),
$$


where

$$
S(T)=\frac{1}{\pi} \operatorname{am} \zeta\left(\frac{1}{2}+i T\right),
$$

the amplitude being obtained by continuous variation along the straight lines joining $2,2+i T$, and $1 / 2+i T$, starting with the value 0 . The variation of $S(T)$ is thus closely related to the distribution of the imaginary parts of the zeros. We know a little about $S(T)$; namely,

$$
S(T)=O(\log T) ; \quad \int_{0}^{T} S(t) d t=O(\log T) ; \quad S(t) \neq o(1) .
$$

Selberg proved the following $\Omega$ result for $S(T)$ :

$$
S(t)=\Omega_{ \pm}\left\{(\log t)^{1 / 3}(\log \log t)^{-7 / 3}\right\} .
$$

Selberg proved some remarkable theorems about the distribution of the zeros of the functions $L(s, \chi)$ where $\chi(n)$ is a character $(\bmod k)$.

If $\left|t_{1}\right| \leqq k^{1 / 4-\epsilon}$ and $A=A(\epsilon)$ is chosen sufficiently great, at least $k / 2$ of the $k-2$ different functions $L(s, \chi)$ (here $k$ is a prime, and the character $\chi$ is nonprincipal) have a zero in the interval described by

$$
\sigma=\frac{1}{2}, \quad t_{1}<t<t_{1}+\frac{A}{\log k} .
$$

Also the total number of zeros of the functions $L(s, \chi)$ in the rectangle $0<\sigma<1, t_{1}<t<t_{1}+A / \log k$ is $O(k)$.

3. On $\zeta(s)$, continued. In some unedited papers left by Riemann (these were in a very untidy state-often with only one side of a formula), Siegel recently found the following result (the proofs were not clear and many details were supplied by Siegel):

$$
\begin{aligned}
\zeta(s)= & \sum_{l=1}^{m} l^{-s}+\frac{(2 \pi)^{s}}{2 \Gamma(s) \cos (s \pi / 2)} \sum_{l=1}^{m} l^{g-1} \\
& +(-1)^{m-1} \frac{(2 \pi)^{(s+1) / 2}}{\Gamma(s)} t^{(s-1) / 2} e^{\pi i s / 2-t i / 2-\pi i / 8} S,
\end{aligned}
$$

with

$$
S=\sum_{0 \leqq 2 r \leqq k \leqq n-1} \frac{2^{-k} i^{r-k} k !}{r !(k-2 r) !} a_{k} F^{(k-2 r)}(\delta),
$$

where 


$$
\begin{gathered}
n \leqq 2 \cdot 10^{-8} t, \quad m=\left[\left(\frac{t}{2 \pi}\right)^{1 / 2}\right], \quad \delta=t^{1 / 2}-\left(m+\frac{1}{2}\right)(2 \pi)^{1 / 2}, \\
F(u)=\frac{\cos \left(u^{2}+3 \pi / 8\right)}{\cos \left((2 \pi)^{1 / 2} u\right)}
\end{gathered}
$$

and the coefficients $a_{k}$ are fixed by the recursion formula

$$
(n+1) \tau a_{n+1}=-(n+1-\sigma) a_{n}+i a_{n-2} \quad\left[a_{-2}=a_{-1}=0\right] .
$$

Here

$$
\tau=t^{1 / 2}, \quad a_{n}=O\left(t^{-n / 2+[n / 3]}\right) .
$$

This formula enables one (theoretically at least!) to obtain rapid approximations to $\zeta(s)$, for example when $\sigma=1 / 2$ and $t$ is large. The principal term of the semi-convergent series for $\zeta(s)$, namely,

$$
\sum_{l=1}^{m} l^{-s}+\frac{(2 \pi)^{s}}{\pi} \sin \frac{s \pi}{2} \Gamma(1-s) \sum_{l=1}^{m} l^{s-1} \quad\left(m=\left[\left(\frac{t}{2 \pi}\right)^{1 / 2}\right]\right),
$$

was also found by Hardy and Littlewood. In place of Riemann's development of $S$, they gave only an upper bound of the absolute value of the "Rest." Siegel says, "Wie stark Riemanns analytische Technik war, geht besonders deutlich aus seiner Ableitung und Uniformung der semikonvergenten Reihe für $\zeta(s)$ hervor."

4. On $\zeta(s)$, continued. We shall now make a few remarks on "Gram's law." These remarks are due to Atle. Selberg. Define

$$
\vartheta=\arg \left\{\pi^{-s / 2} \Gamma\left(\frac{s}{2}\right)\right\}
$$

where

$$
s=\frac{1}{2}+i t
$$

The function $\vartheta(t)$, thus defined, first decreases to a minimum between $-2 \pi$ and $-\pi$ (when $t$ increases from 0 to $\infty$ ) and is from this point onward steadily increasing. For large $t$ we have

$$
\vartheta=\frac{t}{2} \log \frac{t}{2 \pi}-\frac{t}{2}-\frac{\pi}{8}+O\left(t^{-1}\right)
$$

We now for $\nu=0,1,2, \cdots$ determine the positive number $t_{\nu}$ by the conditions $\vartheta^{\prime}\left(t_{\nu}\right)>0$ and 


$$
\vartheta\left(t_{\nu}\right)=(\nu-1) \pi
$$

Then

$$
\zeta\left(\frac{1}{2}+i t_{\nu}\right)=(-1)^{\nu-1} X\left(t_{\nu}\right)
$$

so that $\zeta\left(1 / 2+i t_{v}\right)$ is real.

The Riemann-Siegel formula

gives

$$
X(t)=\sum_{n \leqq(t / 2 \pi)^{1 / 2}} \frac{\cos (\vartheta-t \log n)}{n^{1 / 2}}+O\left(t^{-1 / 4}\right)
$$

$$
\zeta\left(\frac{1}{2}+i t_{\nu}\right)=1+\sum_{1<n<\left(t_{\nu} / 2 \pi\right)^{1 / 2}} \frac{\cos \left(t_{\nu} \log n\right)}{n^{1 / 2}}+O\left(t_{\nu}^{-1 / 4}\right),
$$

which seems to suggest that $\zeta\left(1 / 2+i t_{\nu}\right)$ is generally positive, and that $X\left(t_{\nu-1}\right)$ and $X\left(t_{\nu}\right)$ should generally be of opposite sign, so that $\zeta(1 / 2+i t)$ should generally have at least one real zero in the interval $\left(t_{\nu-1}, t_{\nu}\right)$. The numerical results of Gram and Backlund verified that for $t_{\nu}<200, \zeta\left(1 / 2+i t_{\nu}\right)$ were always positive and that always $t_{\nu-1}$ $<\gamma<t_{\nu}$, so that the $\gamma$ 's were separated by the $t_{\nu}$ 's. It is this property which is referred to as Gram's law.

See A. Selberg, Proceedings of the Tenth Scandinavian Congress, Copenhagen, August, 1946, pp. 197-200. Titchmarsh proved that "Gram's law" has infinitely many exceptions, and Selberg proved that the exceptions even have a positive density; and for this reason Selberg viewed the "Riemann hypothesis" with suspicion.

5. On $\pi(x)$. The equation (5) is fundamental for all estimates of $\pi(x)$, the number of primes not exceeding $x$.

Euclid proved that

$$
\pi(x) \rightarrow \infty \quad[x \rightarrow \infty] .
$$

Legendre and Gauss independently (the latter at a very early age) surmised that

$$
\pi(x) \sim \frac{x}{\log _{e} x}
$$

The sign of asymptotic equality

$$
f(x) \sim g(x)
$$

expresses that 


$$
\lim _{x \rightarrow \infty} \frac{f(x)}{g(x)}=1 .
$$

Riemann, apparently, developed his whole theory of the function $\zeta(s)$ in an attempt to prove the beautiful relation (6), now known as the Prime Number Theorem. He did not succeed, and it was left to Hadamard and de la Vallée Poussin (independently) to give complete and rigorous proofs of (6). These proofs leaned heavily on properties of $\zeta(s)$. Landau simplified these proofs considerably, using essentially Cauchy's theorem and the fact that $\zeta(s) \neq 0$ on the line $\sigma=1$ (or in a region slightly to the left of this line). Wiener proved (6) without using Cauchy's theorem, but used $\zeta(s) \neq 0$ for $\sigma=1$.

It was reserved to Atle Selberg [33] to give a really elementary proof of (6)-a proof which mentions neither the zeta function nor the complex variable. This proof was a great achievement. It rests on Selberg's fundamental lemma, namely,

where

$$
\sum_{p \leqq x} \log ^{2} p+\sum_{p \leqq x} \log p \vartheta\left(\frac{x}{p}\right) \sim 2 x \log x,
$$

$$
\vartheta(x)=\sum_{p \leqq x} \log p .
$$

This result can be proved very simply. From it one can deduce without much difficulty that

$$
\vartheta(x) \sim x
$$

which is equivalent to (6).

Another proof of (6), also based on Selberg's fundamental lemma, was published by Paul Erdös.

6. On $\pi(x)$, continued. Gauss, as well as subsequent workers, noticed that

$$
\pi(x)<\int_{0}^{x} \frac{d u}{\log u}
$$

as far as existing tables went.

Littlewood [25] proved the following deep and beautiful result:

Writing

$$
P(x)=\pi(x)-\int_{0}^{x} \frac{d u}{\log u}
$$

we have 


$$
P(x)=\Omega_{ \pm}\left(\frac{x^{1 / 2} \log \log \log x}{\log x}\right) .
$$

Thus $P(x)$ changes sign infinitely often as $x \rightarrow \infty$. Littlewood's method, which used the Phragmen-Lindelöf principle, does not give an explicit value of $x_{0}$ such that $P\left(x_{0}\right)>0$. Skewes [45] found a method which determines an upper bound for $x_{0}$ such that $P\left(x_{0}\right)>0$, namely,

$$
x_{0}<10^{10^{10^{34}}} .
$$

In this connection see also Littlewood [25] and Turán [51] and Ingham [22].

On the assumption of the Riemann hypothesis one can show that

$$
P(x)=O\left(x^{1 / 2+\bullet}\right)
$$

for every positive $\epsilon$; and conversely, if this relation is true, then the Riemann hypothesis is true.

W. H. Mills [26] found for the first time in 1947 a prime-representing function; he proved the existence of an $A>1$ such that

$$
\left[A^{3^{x}}\right]
$$

is a prime for all positive integers $x$. Here $[x]$ denotes the greatest integer in $x$. This Mills deduced in a trivial way from the fact that there exists a prime between

$$
x^{3} \text { and }(x+1)^{3}
$$

for all large $x$, a result prove by Ingham. Hoheisel was the first to find a number $\theta$ such that

$$
\sum_{x \theta<p<(x+1)^{\theta}} 1>0
$$

for $\theta=33,000$. His $\theta$ was improved successively by Heilbronn, Tchudakoff, Ingham. Whether the above inequality is true for $\theta=2$ is still unknown.

7. Functions allied to $\zeta(s)$. Dirichlet found the remarkable theorem that every arithmetical progression $a x+b(x=1,2,3, \cdots)$ contains an infinity of primes if $a$ and $b$ have no common factor. To prove this classical result he introduced what are known as Dirichlet's $L$-functions. We first define $\chi(n)$, a character $(\bmod k)$, as follows:

$$
\chi(m)=\chi(n) \quad \text { if } m \equiv n(\bmod k) ;
$$




$$
\begin{aligned}
\chi(m n) & =\chi(m) \chi(n) ; \\
\chi(n) & =0 \\
\chi(1) & \neq 0 .
\end{aligned}
$$

Then for $\sigma>1$,

$$
L_{k}(s)=L(s, \chi)=\sum_{1}^{\infty} \chi(n) n^{-s} .
$$

As an example $k=5$ of $L$-functions we take

$$
\begin{aligned}
L_{5}(s)=L(s, \chi)= & 1^{-s}+i \cdot 2^{-s}-i \cdot 3^{-s}-4^{-s}+0 \cdot 5^{-s} \\
& +6^{-s}+i \cdot 7^{-s}-i \cdot 8^{-s}-9^{-s}+0 \cdot 10^{-s} \\
& +11^{-s}+i \cdot 12^{-s}-i \cdot 13^{-s}-14^{-s}+0 \cdot 15^{-s} \\
& +\cdots .
\end{aligned}
$$

In fact the series on the right is convergent for $\sigma>0$. One easily proves

$$
L(s, \chi)=\prod_{p}\left\{1-\chi(p) p^{-8}\right\}^{-1} \quad(\sigma>1) .
$$

For the proof of Dirichlet's theorem it is fundamental that

$$
L(1)=\sum_{1}^{\infty} \chi(n) n^{-1} \neq 0,
$$

where $\chi(n)$ is a character $(\bmod k)$ such that the series is convergent.

The $L$-functions have many properties similar to the zeta function. They can be continued over the whole $s$-plane; they possess functional equations; and the Riemann hypothesis for the $L$-functions simply states that if $L(s, \chi)=0$ for $0<\sigma<1$, then $\sigma=1 / 2$.

It is noteworthy that if one assumes the "extended Riemann hypothesis" (the assumption that the Riemann hypothesis is true for all Dirichlet's $L$-functions), one proves, as Hardy and Littlewood did, that all large odd numbers are expressible as a sum of three odd primes. Vinogradoff proved this result in 1937 without any assumption.

Even the statement

$$
L(s, \chi)>0 \quad(0<s<1)
$$

(here $\chi \neq \chi_{0} ; \chi_{0}$ is the so-called principal character which is such that $\chi_{0}(n)=1$ or 0 for all $n$ ) is still unproved.

Siegel proved that 


$$
\sum_{n=1}^{\infty} \chi(n) n^{-1}>k^{-e}
$$

where $\chi(n)$ is a nonprincipal character $(\bmod k)$, and this shows that $L(s, \chi)>0$ for $1-A(\epsilon) k^{-\epsilon}<s \leqq 1$. Thus the hypothesis (7) is proved only for an infinitesimal portion of the line $0 \leqq s \leqq 1$.

Recently Estermann and Chowla [12] gave new simplified proofs of this result.

Chowla [10] found a method (greatly improved by Rosser [30]) of proving for real nonprincipal characters

$$
L_{k}(s)>0 \quad(0<s<1)
$$

for a large number of special values of $k$. The most difficult case up to $k=200$ was $k=163$. Chowla and Selberg [14] proved that

$$
L_{163}(1 / 2)>0
$$

and Rosser [31] proved that

$$
L_{163}(s)>0
$$

Showalter verified that

$$
L_{409}(s)>0
$$

Regarding $L(1)$ nothing better than the trivial result

$$
L(1)=O(\log k)
$$

is known. This is proved as follows:

$$
\begin{aligned}
L(1) & =\sum_{1}^{\infty} \frac{\chi(n)}{n}=\sum_{1}^{k} \frac{\chi(n)}{n}+\sum_{k+1}^{\infty} \frac{\chi(n)}{n} \\
& =\sum_{1}+\sum_{2} .
\end{aligned}
$$

Since $|\chi(n)|=1$ or 0 ,

$$
\sum_{1}=O(\log k) .
$$

By partial summation using

$$
\left|\sum_{1}^{t} \chi(n)\right| \leqq k,
$$

we get

$$
\sum_{2}=O(1)
$$

Littlewood proved that if $\chi(n)$ is a primitive character $(\bmod k)$, then 


$$
L(1)=O(\log \log k)
$$

on the assumption of the extended Riemann hypothesis. He also proved (again under the assumption of the extended Riemann hypothesis) that there are infinitely many $k$ such that

$$
L(1)<\frac{b}{\log \log k}
$$

where $\chi(n)$ is a real primitive character $(\bmod k)$.

While Linnik and Walfisz proved independently that there are infinitely many $k$ such that

$$
L(1)<\frac{b}{(\log \log k)^{1 / 2}},
$$

Chowla [11] proved Littlewood's result without any assumption.

Bateman, Chowla, and Erdös [6] proved that there are infinitely many primes $p$ for which

$$
L(1)=\sum_{1}^{\infty}\left(\frac{n}{p}\right) n^{-1}<\frac{b}{\log \log p},
$$

where $b$ is a certain suitable positive constant independent of $p$.

8. Functions allied to $\zeta(s)$, continued. Given an algebraic numberfield $K$ we define

$$
\zeta_{K}(s)=\prod_{P}\left\{1-N(P)^{-s}\right\}^{-1},
$$

where $P$ runs over all different prime ideals in $K$, and $N(P)$ denotes the norm of the prime ideal $P$ in $K$.

This function was introduced by Dedekind. Hecke in 1917 proved that

$$
(s-1) \zeta_{K}(s)
$$

can be continued analytically over the whole $s$-plane, and that it is an entire function of $s$.

Further the function $\zeta_{K}(s)$ satisfies a functional equation similar to that satisfied by $\zeta(s)$. See also Siegel [41].

An intriguing conjecture about $\zeta_{K}(s)$ is that the ratio $\zeta_{K}(s) / \zeta(s)$ is an entire function of $s$ for all complex values of $s$. See Artin [5]. The conjecture is known from the work of Takagi to be true when $K$ is an Abelian field over the rationals. Some other cases of the conjecture-non-Abelian cases-were proved by Dedekind and more re- 
cently in the brilliant work of Artin and R. Brauer. Dedekind found for $K=R\left(2^{1 / 3}\right)$ that

$$
\zeta_{K}(s)=\zeta(s)\left\{\sum^{\prime}\left(x^{2}+27 y^{2}\right)^{-s}-\sum^{\prime}\left(4 x^{2}+2 x y+7 y^{2}\right)^{-s}\right\}
$$

where the summations are for all pairs of integers $x, y$ excluding $x=y=0$.

The two expressions in the braces are examples of the so-called Epstein zeta function.

Dedekind's (1900) result had its origin in the following remarks of Gauss. If $p$ is a prime $\equiv 1$ (3) and if 2 is a cubic residue of $p$, then $p=x^{2}+27 y^{2}$. If 2 is not a cubic residue of $p$, then $p=4 x^{2}+2 x y+7 y^{2}$. Gauss stated similar results when 2 is replaced by 3 or 5 .

Hecke (and after him Landau) defined "Abelian $L$-series" as follows. Let $\mathfrak{F}$ be a fixed ideal. Then two ideals $\mathfrak{M}_{1}$ and $\mathfrak{M}_{2}$ are equivalent $(\bmod \mathfrak{F})$ if there exist algebraic integers $\alpha_{1}, \alpha_{2}$ in $K$, such that

$$
\begin{aligned}
\left(\alpha_{1}\right) \mathfrak{M}_{1} & =\left(\alpha_{2}\right) \mathfrak{M}_{2}, \\
\alpha_{1} & \equiv \alpha_{2}(\bmod \mathfrak{F}),
\end{aligned}
$$

provided $\mathfrak{M}_{1}$ and $\mathfrak{M}_{2}$ are prime to $\mathfrak{F}$. It is not difficult to see that all ideals of $K$ fall into a finite number of nonequivalent classes (mod $\mathfrak{F}$ ), and that these nonequivalent classes form a finite multiplicative Abelian group. Let $\chi$ be any character of this group. Then for $\sigma>1$ we define

$$
L(s, \chi)=\prod\left\{1-\chi(P) N(P)^{-s}\right\}^{-1},
$$

where $P$ runs over all different prime ideals of $K$. These Abelian $L$-series can also be continued over the whole $s$-plane. In fact, if $K$ is the cubic field $R\left(d^{1 / 3}\right), d$ an integer $\neq m^{3}$, a striking result of Artin asserts

$$
\zeta_{K}(s)=\zeta(s)\left(L_{1} L_{2}\right)^{1 / 2},
$$

where $L_{1}, L_{2}$ are certain Abelian $L$-series in the field $R\left((-3)^{1 / 2}\right)$. Artin also obtains relations between the zeta functions of certain fields and their subfields. Further the Abelian $L$-series do not vanish for $\sigma=1$ as Hecke found. Further they have a functional equation. They enable us to extend Dirichlet's theorem on primes in an arithmetic progression to the following:

If $(\rho, \mathfrak{F})=1$, then there exist infinitely many integers $\pi$ such that

$$
\pi \equiv \rho(\bmod \mathfrak{F})
$$

where $(\pi)$ is a prime ideal. 
9. Functions allied to $\zeta(s)$, continued. We shall now consider an entirely different type of $L$-series whose introduction into numbertheory has had astounding results. For an account of these see Davenport [15].

Let $f_{1}(x), \cdots, f_{r}(x)$ be any polynomials with integer coefficients, $p$ a prime. We define a normalized polynomial as a polynomial with integer coefficients whose highest coefficient is 1 . Further $(f, g)$, the resultant of $f$ and $g$, is defined as

$$
\text { II } f(\phi),
$$

where $\phi$ runs over the roots of $g(x)=0$.

We then define

$$
L(s)=1+\frac{\sigma_{1}}{p^{s}}+\frac{\sigma_{2}}{p^{2 s}}+\cdots+\frac{\sigma_{K-1}}{p^{(K-1) s}},
$$

where

$$
\sigma_{\nu}=\sum_{g} \chi_{1}\left(\left(f_{1}, g\right)\right) \cdots \chi_{r}\left(\left(f_{r}, g\right)\right),
$$

where the $\chi$ 's are characters $(\bmod p), K=$ sum of degrees of the $f$ 's which are irreducible $(\bmod p)$, and the summation is over all normalized polynomials of degree $\nu$ (in the definition of $\sigma_{\nu}$ above).

These $L$-functions also have a functional equation and a Riemann hypothesis which André Weil proved. However, the proof is exceedingly difficult, and assumes a knowledge of the recent treatment of algebraic geometry which is due to André Weil himself.

In the special case $r=1, f_{1}=x^{3}+a x+b$, we obtain

$$
L(s)=1+\frac{\sigma_{1}}{p^{s}}+\frac{p}{p^{2 s}},
$$

where

$$
\sigma_{1}=\sum_{x=1}^{p}\left(\frac{x^{3}+a x+b}{p}\right) .
$$

Here $\left(\frac{n}{p}\right)$ is Legendre's symbol defined as 0 if $n \equiv 0(p)$, while if $n \neq 0(\bmod p),\left(\frac{n}{p}\right)=1$ or -1 according as the quadratic congruence $x^{2} \equiv n(\bmod p)$ is or is not soluble.

Thus $L(s)=0$ implies

$$
p^{-s}=\frac{\sigma_{1} \pm\left(\sigma_{1}^{2}-4 p\right)^{1 / 2}}{2 p} .
$$


Thus $R(s)=1 / 2$ if and only if

$$
\left|\sigma_{1}\right|<2 p^{1 / 2}
$$

Artin conjectured this in 1924 and verified it in about 40 numerical instances. A proof was given by Hasse in 1934. It uses the complex multiplication of elliptic functions. See also Deuring [17] and Chowla [13].

10. Some unsolved problems. In this section we collect together a few problems of interest whose solution depends on the theory of different types of zeta functions.

(1) Let $k$ be a positve integer; let $f(n)$ have the period $k$, i.e.,

$$
f(m)=f(n) \quad[m \equiv n(\bmod k)] ;
$$

let $f(x)$ be a number-theoretic function; suppose further that not all $f(n)$ are 0 ; then

$$
\sum_{n=1}^{\infty} \frac{f(n)}{n} \neq 0
$$

whenever the series is convergent.

This problem was suggested by Paul Erdös. If true, it naturally includes a result like

$$
\sum_{1}^{\infty}\left(\frac{n}{p}\right) n^{-1} \neq 0
$$

due to Dirichlet. Here $\left(\frac{n}{p}\right)$ is Legendre's symbol. I proved this under the assumptions $f(x)=-f(-x), k=$ a prime, $(k-1) / 2=$ a prime. Professor Siegel, to whom I showed my proof, proved the result under only the first of the above three assumptions, namely, whenever $f(x)=-f(-x)$ is true for all $x$.

REMARK. The condition for the convergence of our series is of course

$$
\sum_{1}^{k} f(n)=0 .
$$

(2) Let $h(d)$ denote the class-number of the quadratic field $R\left(d^{1 / 2}\right)$. It is known that there are nine negative $d$ for which $h(d)=1$,

$$
-d=3,4,7,8,11,19,43,67,163 .
$$

It is also known (Heilbronn and Linfoot) that there is at most one more negative $d$ with $h(d)=1$. This $-d$ must necessarily be a prime $p$ 
of the form $8 k+3$. Chowla and Selberg [14] proved that for such a prime $p$ we would have

$$
\sum_{1}^{\infty}\left(\frac{n}{p}\right) n^{-1 / 2}<0 ;
$$

so that the "extended Riemann hypothesis" would certainly be false!

Another interesting way of stating the conjecture that $h(d)=1$ has no tenth solution with $d<0$ is the following (which I have not seen before).

If $p$ is a prime $\equiv 3(8)$, greater than 163 , then we can write

$$
p=x^{2}+y^{2}+z^{2},
$$

where $x, y, z$ are all different positive integers.

(3) The cosine problem. Let $K$ be an arbitrary positive integer. Then can we find $s_{0}=s_{0}(K)$ such that

$$
\min _{0 \leqq x<2 \pi}\left\{\cos a_{1} x+\cos a_{2} x+\cdots+\cos a_{8} x\right\}<-K
$$

for all $s>s_{0}$ where $a_{1}, \cdots, a_{s}$ is any set of $s$ different positive integers?

This problem is due to Ankeny and Chowla. It has been communicated to several mathematicians, and it appears that the answer is probably in the affirmative, but a proof seems difficult. The problem actually arose in a question concerning zeta functions.

(4) There are infinitely many primes which do not divide a given irreducible polynomial of degree $n \geqq 2$ with integer coefficients.

Professor Siegel, to whom I showed this problem, gave a proof which depends on the theory of the Dedekind zeta function. It does not seem easy to give an "elementary" proof, in particular one which avoids infinite series.

(5) If $f(x)$ is a polynomial with integer coefficients, what is the number of values which $f(x)$ assumes $(\bmod p)$ ?

For example, it is likely that for fixed integers $a, b(a b \not \equiv 0)$

$$
x^{n}+a x+b
$$

assumes more than $p / 2$ values $(\bmod p)$ when $p$ is large enough.

This I have been unable to prove. Professor Davenport, to whom I communicated the problem, obtained the following results:

The number of values assumed by $f(x)=x^{4}+a x^{2}+b x+c(\bmod p)$ is $\sim 5 p / 8$.

(6) If $f(x)=a_{n} x^{n}+\cdots+a_{0}$, where the $a$ 's are integers and $a_{n} \not \equiv 0(p)$, André Weil (see Mordell, Thoughts on number theory, but a detailed proof is still unpublished) proved that 


$$
\left|\sum_{x=1}^{p} e^{2 \pi i f(x) / p}\right| \leqq(n-1) p^{1 / 2} ;
$$

for $n=2$, there is equality (by Gauss).

(7) Is $\zeta(3)$ irrational? This is a well known unsolved problem. For $\zeta(3)$ here one can in general substitute $\zeta(2 n+1)$ where $n$ is any positive integer.

(8) Heuristically one can argue (as Artin did; see Bilharz [7]) that the primes for which 2 is a primitive root have an exact positive density. However, even assuming any of the Riemann hypotheses one has not been able to prove this.

(9) While the usual expression for the class-number of a real quadratic field contains infinite series or transcendental functions, the following expression (due to Ankeny, Artin, and Chowla; see [1]) for the class-number of a real quadratic field is purely arithmetical:

$$
2 h \frac{u}{t} \equiv \frac{A+B}{p}(\bmod p) .
$$

Here $h$ is the class-number of the real quadratic field $R\left(p^{1 / 2}\right)$, where $p$ is a prime $\equiv 1(\bmod 4)$,

$$
\epsilon=\left(t+u p^{1 / 2}\right) / 2
$$

is its fundamental unit; $A$ stands for the product of the least positive quadratic residues of $p, B$ for the corresponding product of nonresidues.

Let $q$ be a prime $\equiv 7(\bmod 12), H$ the class-number of the imaginary quadratic field $R\left((-q)^{1 / 2}\right), h$ the class-number of the real quadratic field $R\left((3 q)^{1 / 2}\right), \epsilon=\left(t+u(3 q)^{1 / 2}\right) / 2$ the fundamental unit of the latter field.

Then (see [1])

$$
H \equiv-\frac{u}{t} h
$$

(10) Is

$$
R L(1)>0,
$$

where

$$
L(1)=\sum_{1}^{\infty} \chi(n) n^{-1}
$$


and $\chi(n)$ is a complex character $(\bmod k)$ ? This problem was raised by Chowla and verified by Showalter in many cases.

(11) Pillai's problem. If $p_{n}$ is the $n$th prime, is it true that

$$
\left|\sum_{n \leqq x}(-1)^{n-1} p_{n}\right| \sim \frac{1}{2} p_{x}
$$

where $x \rightarrow \infty$ through positive integral values?

(12) Is it true that

$$
\sum_{1}^{t} \chi(n) n^{-1}>0
$$

if $\chi(n)$ is a real nonprincipal character $(\bmod k)$, and $t$ is an arbitrary number?

(13) If $1^{-s}+2^{-s}+\cdots+n^{-s} \neq 0 \quad\left(\sigma>1, n>n_{0}\right)$ then the "Riemann hypothesis" is true. This result is due to Turán [52].

(14) If $h(d)$ is the class-number of the quadratic field $R\left(d^{1 / 2}\right)$, Gauss conjectured that

$$
h(d)=1
$$

has infinitely many solutions with $d>0$. This problem remains far from solution. See K. Schaffstein, Math. Ann. vol. 98 (1928). In particular Schaffstein finds primes $p>10^{6}$ with $h(p)=1$.

\section{BIBLIOGRAPHY}

1. N. C. Ankeny, E. Artin, and S. Chowla, On the class number of real quadratic fields, Proc. Nat. Acad. Sci. U.S.A. vol. 37 (1951) pp. 524-525.

2. N. C. Ankeny and S. Chowla, On the class number of the cyclotomic field, Proc. Nat. Acad. Sci. U.S.A. vol. 35 (1949) pp. 529-532 and Canadian Journal of Mathematics vol. 3 (1951) pp. 486-494.

3. N. C. Ankeny and C. A. Rogers, On a conjecture of Chowla, Ann. of Math. (2) vol. 53 (1951) pp. 541-550.

4. E. Artin, Über eine neue Art von L-Reihen, Abh. Math. Sem. Hamburgischen Univ. vol. 3 (1923) pp. 89-108.

5. - Über die zeta Funktion gewisser algebraischer Zahlkörper, Math. Ann. vol. 89 (1923) pp. 147-156.

6. P. Bateman, S. Chowla, and P. Erdös, Remarks on the size of $L(1, \chi)$, Publicationes Mathematicae Debrecen vol. 1 (1950) pp. 165-182.

7. H. Bilharz, Primdivisoren mit vorgegebener Primitivwurzel, Math. Ann. vol. 114 (1937) pp. 476-492.

8. R. Brauer, On the zeta functions of algebraic number fields, Amer. J. Math. vol. 69(1947) pp. 243-250.

9. - On Artin's L-series with general group characters, Ann. of Math. vol. 48 (1947) pp. 502-514.

10. S. Chowla, Note on Dirichlet's L-functions, Acta Arithmetica vol. 1 (1935) pp. 113-114. 
11. - Improvement of a theorem of Linnik and Walfisz, Proc. London Math. Soc. (2) vol. 50 (1949) pp. 423-429.

12. - A new proof of a theorem of Siegel, Ann. of Math. (2) vol. 51 (1950) pp. $120-122$.

13. - On the last entry in Gauss's Diary, Proc. Nat. Acad. Sci. U.S.A. vol. 35 (1949) pp. 244-246.

14. S. Chowla and A. Selberg, On Epstein's zeta function (I), Proc. Nat. Acad. Sci. U.S.A. vol. 35 (1949) pp. 371-374.

15. H. Davenport, On character sums in finite fields, Acta Math. vol. 71 (1939) pp. $99-121$.

16. R. Dedekind, Ueber die Anzahl der Idealklassen in reinen kubischen Zahlkörpern, J. Reine Angew. Math. vol. 121 (1900) pp. 40-123.

17. M. Deuring, Die Typen der Multiplikatorenringe elliptischer Funktionenkörper, Abh. Math. Sem. Hansischen Univ. vol. 14 (1941) pp. 197-272.

18. E. Hecke, Eine neue Art von Zetafunktionen und ihre Beziehungen zur Verteilung der Primzahlen, Math. Zeit. vol. 1 (1918) pp. 357-376.

19. - Eine neue Art von Zetafunktionen und ihre Beziehungen zur Verteilung der Primzahlen (Zweite Mitteilung), Math. Zeit. vol. 6 (1920) pp. 11-51.

20. A. E. Ingham, On the difference between consecutive primes, Quart. J. Math. Oxford Ser. vol. 8 (1937) pp. 255-266.

21. - On the estimation of $N(\sigma, T)$, Quart. J. Math. Oxford Ser. vol. 11 (1940) pp. 291-292.

22. - A note on the distribution of primes, Acta Arithmetica vol. 1 (1936) pp. 201-211.

23. E. Landau, Über die Wurzeln der Zetafunktion eines algebraischen Zahlkörpers, Math. Ann. vol. 79 (1919) pp. 388-401.

24. —— Über einige ältere Vernutungen und Behauptungen in der Primzahltheorie, Math. Zeit. vol. 1 (1918) pp. 1-24.

25. J. E. Littlewood, Mathematical Notes (1 2): An inequality for a sum of cosines, J. London Math. Soc. vol. 12 (1947) pp. 217-221.

26. W. H. Mills, $A$ prime representing function, Bull. Amer. Math. Soc. vol. 53 (1947) pp. 604, 1196.

27. L. J. Mordell, Thoughts on number theory, J. London Math. Soc. vol. 21 (1946) pp. 58-74.

28. Balth. van der Pol, An electro-mechanical investigation of the Riemann zeta function in the critical strip, Bull. Amer. Math. Soc. vol. 53 (1947) pp. 976-981.

29. A. Renyi, On the large sieve of Ju. V. Linnik, Compositio Math. vol. 8 (1950) pp. 68-75.

30. J. B. Rosser, Real roots of Dirichlet L-series, Bull. Amer. Math. Soc. vol. 55 (1949) pp. 906-913.

31. - Real zeros of real Dirichlet L-series, unpublished.

32. K. Schaffstein, Tafel der Klassenzahlen der reelen quadratischen Zahlkörper mit Primzahldiskriminanten unter 12,000 und zwischen 100,000-101,000 und 1,000,0001,001,000, Math. Ann. vol. 98 (1928) pp. 745-748.

33. A. Selberg, An elementary proof of the prime number theorem, Ann. of Math. (2) vol. 50 (1949) pp. 305-313.

34. - Contributions to the theory of Dirichlet's L-functions, Skrifter Utgitt av Det Norske Videnskaps-Akademi i Oslo. I Mat.-Naturv. Klasse 1946, No. 3.

35. - On the normal density of primes in small intervals, and the difference 
between consecutive primes, Archiv for Mathematik og Naturvidenskab vol. 47, No. 6, pp. 1-19.

36. , Contributions to the theory of the Riemann zeta function, Archiv for Mathematik og Naturvidenskab vol. 48, No. 5.

37. - On the zeros of Riemann's zeta function, Skrifter Utgitt av Det. Norske Videnskaps-Akademi i Oslo. I Mat.-Naturv. Klasse, 1942, No. 10, pp. 1-59.

38. - The zeta function and the Riemann hypothesis, Comptus Rendus du Dixième Congrès des Mathématiciens Scandinaves tenu à Copenhague, 1946, pp. 26-30.

39. - See S. Chowla and A. Selberg (14 above).

40. - On an elementary method in the theory of primes, Det. Kongelige Norske Videnskabers Selskabs Forhandlinger vol. 19 (1947) No. 18.

41. C. L. Siegel, Neuer Beweis für die Funktionalgleichung der Dedekindschen zeta Funktion, Math. Ann. vol. 85 (1922) pp. 123-128.

42. - Neuer Beweis fiur die Funktionalgleichung der Dedekindschen zeta Funktion, Nachr. Ges. Wiss. Göttingen (1922) pp. 25-31.

43. - Über die Klassenzahl algebraischer Zahlkörper, Acta Arithmetica vol. 1 (1935) pp. 83-86.

44. - Ü̈ber Riemann's Nachlass zur analytischen Zahlentheorie, Quellen and Studien z. geschichte d. Mathematik vol. 2 (1932) pp. 45-80.

45. S. Skewes, On the difference $\pi(X)-\mathrm{Li}(X)$, J. London Math. Soc. vol. 8 (1933) pp. 277-283.

46. N. G. Tchudakoff, On the zeros of the function $\zeta(s)$, C. R. Acad. Sci. URSS. vol. 1 (1936) pp. 201-204.

47. E. C. Titchmarsh, On van der Corput's method and the zeta function of Riemann IV, Quart. J. Math. Oxford Ser. vol. 5 (1934) pp. 90-105.

48. - - The zeros of the Riemann zeta function, Proc. Roy. Soc. London Ser. A vol. 151 (1935) pp. 234-255.

49. - The zeros of the Riemann zeta function, Proc. Roy. Soc. London Ser. A vol. 157 (1936) pp. 261-263.

50. —— On $\zeta(s)$ and $\pi(x)$, Quart. J. Math. Oxford Ser. vol. 9 (1938) pp. 97108.

51. P. Turán, On a theorem of Littlewood, J. London Math. Soc. vol. 21 (1946) pp. $268-275$.

52. - On some approximative Dirichlet polynomials in the theory of the zeta function of Riemann, Det. Kgl. Danske Videnskabernes Selskab Matematisk-Fysiske Medellelser. vol. 24 (1948) No. 17.

UNIVERSITY OF KANSAS 